\title{
Physical exercise for late-life major depression
}

\author{
M. Belvederi Murri, M. Amore, M. Menchetti, G. Toni, F. Neviani, M. Cerri, M. B. L. Rocchi, \\ D. Zocchi, L. Bagnoli, E. Tam, A. Buffa, S. Ferrara, M. Neri, G. S. Alexopoulos, S. Zanetidou \\ and the Safety and Efficacy of Exercise for Depression in Seniors (SEEDS) Study Group
}

\section{Background}

Interventions including physical exercise may help improve the outcomes of late-life major depression, but few studies are available.

\section{Aims}

To investigate whether augmenting sertraline therapy with physical exercise leads to better outcomes of late-life major depression.

\section{Method}

Primary care patients ( $>65$ years) with major depression were randomised to 24 weeks of higher-intensity, progressive aerobic exercise plus sertraline (S+PAE), lowerintensity, non-progressive exercise plus sertraline (S+NPE) and sertraline alone. The primary outcome was remission (a score of $\leqslant 10$ on the Hamilton Rating Scale for Depression).

\section{Results}

A total of 121 patients were included. At study end, $45 \%$ of participants in the sertraline group, 73\% of those in the S+NPE group and $81 \%$ of those in the S+PAE group achieved remission $(P=0.001)$. A shorter time to remission was observed in the S+PAE group than in the sertraline-only group.

\section{Conclusions}

Physical exercise may be a safe and effective augmentation to antidepressant therapy in late-life major depression.

\section{Declaration of interest}

None.

\section{Copyright and usage}

(c) The Royal College of Psychiatrists 2015.
Late-life major depression is associated with individual suffering, increases in medical morbidity and mortality, ${ }^{1-3}$ healthcare service use and costs for society. ${ }^{4}$ Available antidepressant therapies currently provide unsatisfactory results, as no more than half of those treated reach remission after a single treatment course. ${ }^{5}$ Therefore, any viable and safe strategy that can improve the efficacy of antidepressant drugs will critically contribute to the management of late-life depression. ${ }^{6}$ It has been proposed that physical exercise could improve the effectiveness of pharmacological treatments in geriatric major depression. ${ }^{7,8}$ In fact, physical exercise can counteract key biological alterations linked with depression that are only partially modified by antidepressant drug therapy: ${ }^{9,10}$ it increases levels of neurotrophic factors, including brain-derived neurotrophic factor (BDNF), and neurogenesis, leading to improvements in neurocognitive performance; ${ }^{11,12}$ exercise also reduces pro-inflammatory cytokines and hypothalamic-pituitary-adrenal (HPA) axis activity. ${ }^{13,14}$ Moreover, physical exercise counteracts disability and social isolation, ${ }^{15}$ while improving psychological self-evaluation. ${ }^{13}$ So far, no study has tested such a hypothesis or examined whether the intensity of physical exercise influences its antidepressant effect, as it has been observed in younger adults. ${ }^{16}$ Our aim was therefore to investigate whether the combination of sertraline treatment with two types of physical exercise - higher-intensity, progressive aerobic exercise and lower-intensity, non-progressive physical exercise - could lead to better outcomes in late-life major depression over 24 weeks than sertraline alone. The study was conducted with participants more than 65 years old who had major depression, with a recruitment strategy based on primary care, where most cases of depression are usually seen and treated. ${ }^{4}$

\section{Method}

The Safety and Efficacy of Exercise for Depression in Seniors (SEEDS) study was a single-blind, randomised study that included 121 patients from four centres in the region of Emilia Romagna,
Italy (Bologna East, Bologna West, Parma and Modena-Correggio). Of these, two were university departments and two were community mental health centres. Recruitment took place in the context of a regional liaison programme between the mental health and primary care departments. The study was conducted in accordance with the principles of the Declaration of Helsinki; eligible patients provided written informed consent before participating.

\section{Recruitment}

The recruitment phase was preceded by meetings with primary care physicians to present the study, providing training on diagnosis of depression and selection criteria. The physicians were asked to select from subsequent visits patients who complained of depressive symptoms and whose health status was compatible with regular exercise. Study coordinators at each centre monitored recruitment through regular contact with the physicians (at least once every 6 weeks) during which they discussed logistics including recruitment and scheduling of visits. The following inclusion criteria were verified during the selection visit by research-oriented psychiatrists: age 65-85 years; a diagnosis of major depressive disorder; a score on the 17-item Hamilton Rating Scale for Depression (HRSD) of 18 or higher; ${ }^{17}$ and being sedentary (not meeting the recommended levels of physical activity for older adults). ${ }^{18}$ Exclusion criteria were other Axis I diagnoses, substance or alcohol misuse, cognitive impairment, defined as a Mini Mental State Examination (MMSE) score of less than $24,{ }^{19}$ and the presence of severe or unstable physical illness that would prevent exercise (e.g. unstable angina, arrhythmias, severe osteoarthritis with functional limitations, uncontrolled diabetes, New York Heart Association class III heart failure, Parkinson's disease, severe respiratory disease). The second visit was then programmed with the study cardiologist to assess aerobic capacity under electrocardiographic monitoring. Rest and peak maximum oxygen uptake $\left(\mathrm{VO}_{2} \max \right)$ was tested on a cycle ergometer (Corival, Lode Medical Technology, Groningen, The Netherlands) with breath-by-breath expired gas analysis (Oxycon 
Pro Metabolimeter, CareFusion, Yorba Linda, California, USA). The test had a duration of 12 min: it began with a 3 min warm-up and was carried out with workload increments of $10 \mathrm{~W} / \mathrm{min}$ for women and $15 \mathrm{~W} / \mathrm{min}$ for men. The test was interrupted earlier than $12 \mathrm{~min}$ in the following conditions: muscular exhaustion, refusal of the patient to continue, respiratory quotient $\geqslant 1.03$, reaching the plateau of oxygen consumption. ${ }^{20}$ The test was suspended and the participant excluded on appearance of signs of atrial fibrillation or other arrhythmias.

\section{Randomisation and masking}

Randomisation was performed after the cardiologist visit by computer with a randomised permutation blocks method (size of blocks 4), stratifying individuals according to study centre, severity of depression (Clinical Global Impression scale severity item mildly/moderately $v$. severely ill), ${ }^{21}$ past treatments with antidepressant (yes $v$. no), age ( $<75$ years $v$. $\geqslant 75$ years) and gender. Each study centre coordinator was sent a personal email containing participants' group assignments and was allowed to share this information only with the study instructors, while keeping the clinical assessor unaware of participant allocation. Also, participants were asked not to disclose to the clinical assessors the type of intervention they were receiving.

\section{Interventions}

Participants were randomly assigned to receive sertraline only $(n=42)$, sertraline plus supervised group non-progressive exercise $(n=37)$ or sertraline plus supervised group progressive aerobic exercise $(n=42)$

\section{Sertraline}

Patients in the sertraline-only condition were prescribed the drug by the centre psychiatrists, with a slow titration scheme to reach the standard dosage of $50 \mathrm{mg}$ within 2 weeks. The choice of sertraline was based on its safety and efficacy in elderly patients and on its low potential for pharmacological interactions. ${ }^{22}$ The prescribing dosage was decided on according to clinical response and the presence of side-effects, in accordance with routine clinical practice. For insomnia, daily use of a hypnotic (zolpidem up to $10 \mathrm{mg}$ /day or lorazepam up to $2 \mathrm{mg}$ /day) was allowed during the first 4 weeks of treatment and discouraged later. At each visit participants were asked to bring their medications to verify their adherence to the prescription scheme. Episodes of non-adherence, defined as not taking the prescribed dosage for at least 3 consecutive days, were recorded.

\section{Sertraline plus non-progressive exercise}

Participants in the sertraline plus non-progressive exercise (S+NPE) arm received sertraline as in the sertraline-only group. In addition they were prescribed attendance at three supervised group exercise sessions per week (60 min duration) for 24 weeks in groups of three to six participants. These sessions were designed to improve participants' strength, balance, respiration and motor coordination, and comprised both mat work and instrumental exercises (see Appendix). Sessions were conducted using heartrate monitors with on-screen visualisation (Polar Team, Polar Electro, Woodbury, New York, USA). Participants in this group were assigned to exercise at heart rate ranges designed not to exceed $70 \%$ of their peak rate. Participants who exceeded their established peak heart rate were asked by the instructor to reduce the intensity of the exercise until their heart rate recovered.

\section{Sertraline plus progressive aerobic exercise}

Participants assigned to sertraline plus progressive aerobic exercise (S+PAE) received sertraline as in the sertraline-only study arm. The schedule of exercise sessions overlapped with those of S+NPE group (three 60 min sessions per week for 24 weeks, in groups of three to six participants). The exercises were mainly based on the use of exercise bicycles, aimed at improving cardiopulmonary condition. ${ }^{23,24}$ Each session began with a 10 min warm-up breathing exercise, followed by cycling at an intensity that would maintain the heart rate within the assigned training range ( $60 \%$ of peak heart rate). Exercise intensity was monitored by the instructor using heart-rate meters as in the $\mathrm{S}+\mathrm{NPE}$ arm. The training scheme was programmed to increase over the weeks, adapting to possible increases in peak heart rate, and to include brief sessions of interval training. All exercise sessions concluded with 5-10 min of cool-down cycling (see Appendix).

\section{Assessments}

Psychiatric diagnoses were assigned by study psychiatrists after administration of the Mini International Neuropsychiatric Interview (MINI). ${ }^{25}$ Assessments took place at baseline and after 4 weeks, 8 weeks, 12 weeks and 24 weeks (within no more than 3 days of the scheduled visit): they included a physical examination, general and psychiatric history, administration of the MMSE, the Cumulative Illness Rating Scale (CIRS) ${ }^{26}$ MINI, HRSD, Clinical Global Impression (CGI) scale and the International Physical Activity Questionnaire (IPAQ). ${ }^{27}$ Assessors were already extensively trained in the use of these instruments; however, four meetings were held to improve interrater reliability on a set of five illustrated cases. Participants who refused to undertake any further assessment with the study personnel were considered to have left the study.

\section{Outcomes}

The primary outcome of this study was the rate and time to remission from depression over the 24 -week period, with remission defined as an HRSD score of 10 or below; this scale rates symptoms and signs of depression occurring during the week preceding its administration. Secondary outcomes were changes in severity of depression (continuous HRSD scores), global improvement of depression (CGI) and changes in aerobic capacity.

\section{Statistical analysis}

A post hoc power analysis showed that a sample size of 40 patients per intervention arm (120 participants) would give an $85 \%$ power to detect a $30 \%$ difference in remission rates $(35 \%$ v. $65 \%$, $P<0.05)$ and a $92 \%$ power to detect a standardised mean difference of 0.34 in HRSD scores $(P<0.05)$, similar to the effect yielded by a recent meta-analysis. ${ }^{7}$ We aimed at assessing the superiority of each combined intervention (S+NPE and S+PAE) compared with antidepressant drug treatment for remission rates, time to remission and other secondary outcomes. To estimate differences in the time to achieve remission we used a survival analysis with Kaplan-Meier estimates of the survival function. A Cox regression analysis was then performed to test whether results held after adjustment for demographic and clinical confounders. To analyse the patterns of change in depression scores over time we performed multilevel hierarchical growth curve analysis using repeated HRSD scores as the dependent variable (baseline, 4 weeks, 8 weeks, 12 weeks and 24 weeks), nested within individual and study centre. The linear, quadratic and cubic terms of time were 
also tested as fixed parameters, along with the interaction terms with group. ${ }^{28}$ Estimation of the parameters was done using the maximum likelihood method and heterogeneous autoregressive covariance structure. Analyses were carried out in the intentionto-treat population, using the last observation carried forward (LOCF) method to impute missing data. All analyses were performed using SPSS version 15.0 on Windows 7.

\section{Results}

Eligible participants were recruited from January 2011 to June 2012. A total of 121 patients entered the study and were randomised to the three treatments: 42 in the sertraline group, 37 in the $\mathrm{S}+\mathrm{NPE}$ group and 42 in the S+PAE group (Fig. 1). Most participants were recruited in the Bologna East centre (sertraline $n=18, \mathrm{~S}+\mathrm{NPE} n=17, \mathrm{~S}+\mathrm{PAE} n=18$ ), smaller numbers were drawn from Bologna West (sertraline $n=11$, S+NPE $n=10$, S+PAE $n=9$ ), Parma (sertraline $n=10$, S+NPE $n=4, \mathrm{~S}+\mathrm{PAE}$ $n=9$ ) and Modena-Correggio (sertraline $n=3$, S+NPE $n=6$, S+PAE $n=6$ ). Participants' demographic and clinical characteristics are reported in Table 1. Their mean age was 75 years (s.d. $=6$ ); the majority were women, most were not married and had elementary or lower educational level. The most frequent physical comorbidities were hypertension (64\%), musculoskeletal problems (61\%), metabolic-endocrinological disorders (40\%) and conditions relating to eye, ear, nose and throat (39\%). The median HRSD score for the whole sample was 18 (interquartile range (IQR) 18-22), indicating mild to moderate severity of depressive symptoms; most participants had already been treated with antidepressant drugs at some point in their lives - more than $80 \%$ with a selective serotonin reuptake inhibitor (SSRI).

\section{Attrition, safety and adherence}

A few people withdrew from the study: six from the sertraline only arm, five from the S+NPE arm and four from the S+PAE arm $\left(14 \%, 14 \%\right.$ and $10 \%$ respectively; $\chi^{2}=0.50$, d.f. $\left.=2, P=0.78\right)$.
Reasons for withdrawing were unwillingness to remain in the study $(n=10)$, new medical problems $(n=4)$ and the need for higher level of care for depression $(n=1)$. Common mild side-effects attributable to the medication included nausea, diarrhoea and insomnia and were rare. Two people left the study because of incidents correlated with physical exercise (one in the S+NPE group and one in the S+PAE group), one for ankle distortion and the other for worsening of musculoskeletal pain. The majority of participants attended assessment visits, with no significant between-group difference (sertraline 79\%, S+NPE 78\%, S+PAE $81 \%$; $\chi^{2}=0.10$, d.f. $=2, P=0.95$ ). All participants received $50 \mathrm{mg}$ of sertraline at entry. By the study end $43 \%$ of those in the sertraline-only group had their dosage increased by the study psychiatrists, as opposed to $22 \%$ in the S+NPE group and $17 \%$ in the S+PAE group $\left(\chi^{2}=8,121\right.$, d.f. $=2, P=0.02$; pairwise comparisons $P<0.05)$. The rate of non-adherence to sertraline treatment tended to be higher in those in the drug-only arm $(26 \%)$ than in the combined interventions (S+PAE 7\%, S+NPE $16 \%, \chi^{2}=5.53$, d.f. $\left.=2, P=0.06\right)$. Attendance at exercise sessions was high ( $70 \%$ for 72 total sessions) and similar in the two exercise groups (S+PAE median 54 sessions, IQR 44-64; S+NPE median 51 sessions, IQR 38-58; Mann-Whitney $z=2.167$, $P=0.14$ ), with a tendency to decrease over the follow-up period.

\section{Remission of depression}

Remission rates were higher in the S+PAE and S+NPE groups than in the sertraline-only group: at week 4 , rates were $36 \%$, $40 \%$ and $7 \%$ respectively $(P=0.001)$. At week 8 , rates were $60 \%, 49 \%$ and $40 \%(P=0.22)$ and at week 12 they were $83 \%$, $54 \%$ and $45 \%(P=0.001)$. By the study end, remission was achieved by $45 \%$ of participants in the sertraline group, $73 \%$ of those in the S+NPE group and $81 \%$ of those in the S+PAE group $(P=0.001)$. Figure 2 reports survival curves for the three intervention groups; in Kaplan-Meier analyses time to remission was significantly different between groups (log rank $\chi^{2}=12.6$, d.f. $=2, P=0.002$ ): this was driven by a shorter time to remission

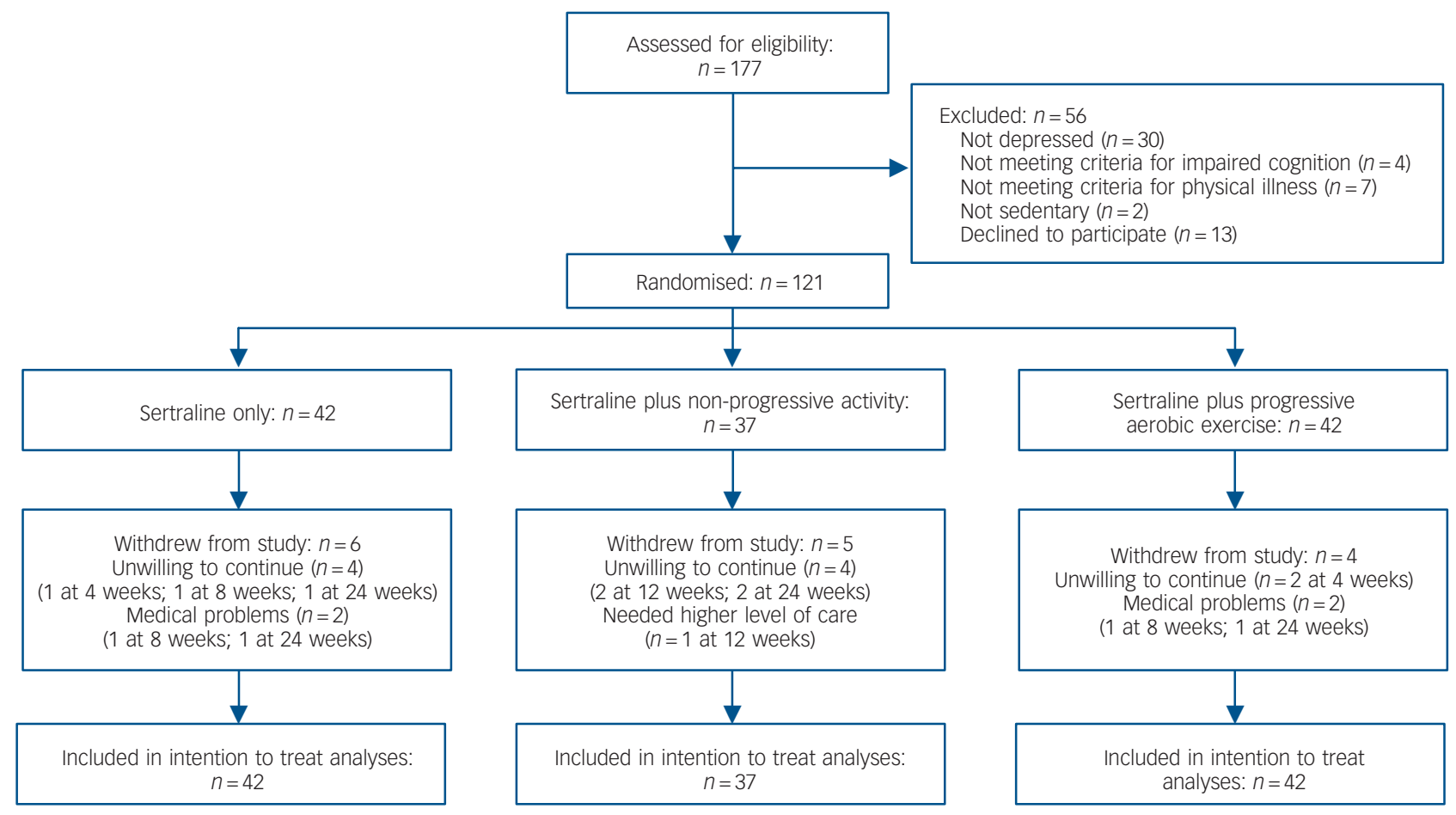

Fig. 1 Study profile 


\begin{tabular}{|c|c|c|c|}
\hline & $\begin{array}{l}\text { Sertraline group } \\
\qquad(n=42)\end{array}$ & $\begin{array}{l}\text { S+NPE group } \\
\quad(n=37)\end{array}$ & $\begin{array}{l}\text { S+PAE group } \\
(n=42)\end{array}$ \\
\hline Age, years: mean (s.d.) & $75.6(5.6)$ & $75.0(6.3)$ & $75.0(6.2)$ \\
\hline Female, $n(\%)$ & $32(76)$ & $25(68)$ & $29(69)$ \\
\hline \multicolumn{4}{|l|}{ Marital status, $n(\%)$} \\
\hline Married & $19(45)$ & $18(49)$ & $18(43)$ \\
\hline Widowed & $19(45)$ & $13(35)$ & $19(45)$ \\
\hline Education: elementary or less, $n(\%)$ & $27(64)$ & $18(49)$ & $20(48)$ \\
\hline Living alone, $n(\%)$ & $19(45)$ & $17(46)$ & $18(43)$ \\
\hline BMI, kg/m²: mean (s.d.) & $25.8(3.3)$ & $25.2(3.7)$ & $26.7(3.8)$ \\
\hline \multicolumn{4}{|l|}{ CIRS } \\
\hline Severity index: mean (s.d.) & $1.23(1.15-1.48)$ & $1.38(1.27-1.62)$ & $1.31(1.21-1.67)$ \\
\hline Comorbidity index: mean (s.d.) & $0(0-1)$ & $1(1-3)$ & $1(0-2)$ \\
\hline Low IPAQ level of physical activity, $n$ (\%) & $34(81)$ & 35 (95) & $35(83)$ \\
\hline$V_{O_{2}}$ max peak, ml/kg per min: mean (s.d.) & $15.3(2.5)$ & $14.8(3.0)$ & $15.2(3.7)$ \\
\hline MMSE total score: mean (s.d.) & $26.8(2.5)$ & $26.8(2.4)$ & $27.0(2.3)$ \\
\hline \multicolumn{4}{|l|}{ HRSD total score: median (IQR) } \\
\hline Baseline & $19(18-23)$ & $18(18-22)$ & $18(18-21)$ \\
\hline 4 weeks & $15.5(12-19.3)$ & $13(8-17.5)$ & $12.5(9-14.3)$ \\
\hline 8 weeks & $13(7.75-17.3)$ & $11(7-13)$ & $9.5(6.75-13)$ \\
\hline 12 weeks & $11.5(8-16)$ & $10(6-13)$ & $8(5.75-10)$ \\
\hline 24 weeks & $11.5(7-16.3)$ & $8(5-11)$ & $7(4-10)$ \\
\hline Age at onset of depressive disorder, years: mean (s.d.) & $48.7(22.7)$ & $50.0(23.5)$ & $49.5(24.3)$ \\
\hline Treated with antidepressant (lifetime), $n$ (\%) & $31(74)$ & $26(70)$ & $25(60)$ \\
\hline More than 1 depressive episode (lifetime), $n$ (\%) & $25(60)$ & $18(49)$ & $20(48)$ \\
\hline Life events in 12 months before interview, $n$ (\%) & $27(64)$ & $16(43)$ & $19(45)$ \\
\hline History of suicide attempt, $n(\%)$ & $1(2)$ & $1(3)$ & $1(2)$ \\
\hline
\end{tabular}

in the S+PAE group (9.3 weeks, 95\% CI 7.4-11.2) than in the sertraline group (14.8 weeks, 95\% CI 12.2-17.4), whereas the time to remission was intermediate for the S+NPE group (12.0 weeks, 95\% CI 9.2-14.8). Compared with participants in the drug-only group, those in the S+PAE group had a twofold hazard ratio for an earlier remission; this value survived after adjusting for various study contextual and clinical factors (Table 2). Furthermore, the hazard ratio for the S+NPE group also became statistically significant with a similar value (1.91) when the same factors were taken into account.

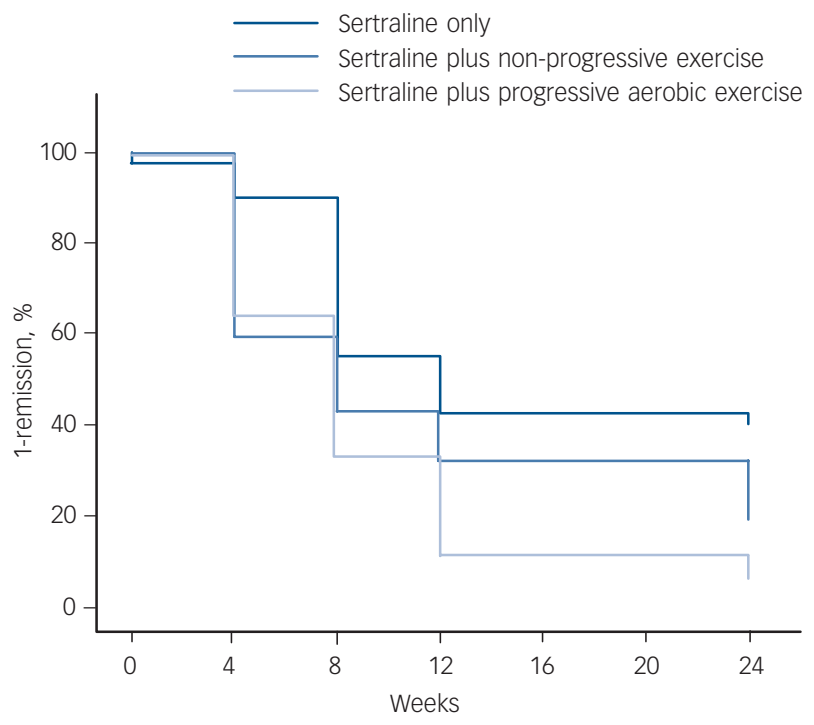

Fig. 2 Kaplan-Meier survival curves for remission of depression according to intervention group.

\section{Changes in depressive symptoms}

There were greater decreases in HRSD scores in the two exercise groups compared with the drug-only group (Table 3, Fig. 3). The overall time course of HRSD scores was best predicted by a third-order polynomial of time, and by the interactions of intervention group (S+NPE $v$. sertraline and S+PAE $v$. sertraline) with time (Table 4). This model's fitness showed significant improvements over models including only the linear and quadratic terms of time (both $2 \mathrm{LL}$ decreases $>45$, d.f. $=3$, $P<0.01$ ), whereas adjusting for the control variables did not lead to further improvement in the model fitness. There was no significant association between the baseline severity of depression and the rate of decrease over time (rho: $P=0.15$ ). Last, the percentage of participants rated as 'very much improved' or 'much improved' on the CGI (scores 1 and 2) at the study end was significantly higher in the S+PAE group $(71 \%)$ and in the S+NPE group $(60 \%)$ than in the sertraline-only group $(43 \%$; $\chi^{2}=7.09$, d.f. $=2, P=0.03$ )

\section{Changes in aerobic capacity}

Only 73 participants had their aerobic capacity assessed at both baseline and 24 weeks (25 in the S+NPE group, 34 in the $\mathrm{S}+\mathrm{PAE}$ group and 14 in the sertraline group). Peak $\mathrm{VO}_{2}$ was on average stable among participants in the S+NPE group $(-0.22 \%$; IQR -14.5 to -12.9$)$, whereas those in the S+PAE group showed a median improvement of $4.7 \%$ (IQR -10.3 to $16.8)$ and those in the sertraline-only group a decrease $(-2.5 \%$, IQR -18.6 to -5.9$)$. However, after adjusting for baseline peak aerobic capacity, intervention group did not predict aerobic capacity at 24 weeks ( $\mathrm{S}+\mathrm{NPE}$ group $P=0.22$, S $+\mathrm{PAE}$ group $P=0.32)$, whereas the number of sessions attended in both groups did $(P=0.03)$. 


\begin{tabular}{|c|c|c|c|}
\hline Predictor & B (s.e.) & $\mathrm{HR}$ & $95 \% \mathrm{Cl}$ \\
\hline \multicolumn{4}{|l|}{ Model $1^{\mathrm{a}, \mathrm{b}}$} \\
\hline S+NPE & $0.51(0.27)$ & 1.68 & $0.98-2.88$ \\
\hline $\mathrm{S}+\mathrm{PAE}$ & $0.75(0.26)$ & $2.12^{* *}$ & $1.27-3.54$ \\
\hline \multicolumn{4}{|l|}{ Model $2^{a, c}$} \\
\hline S+NPE & $0.65(0.29)$ & $1.91 *$ & $1.08-3.39$ \\
\hline S+PAE & $0.72(0.29)$ & $2.04^{\star}$ & $1.17-3.58$ \\
\hline \multicolumn{4}{|l|}{ Centre } \\
\hline Bologna East & $-0.69(0.30)$ & $0.50 *$ & $0.28-0.89$ \\
\hline Parma & $0.44(0.37)$ & 1.56 & $0.76-3.21$ \\
\hline Modena-Correggio & $-0.38(0.40)$ & 0.68 & $0.31-1.50$ \\
\hline \multicolumn{4}{|l|}{ Clinical factors } \\
\hline Age & $<0.01(0.02)$ & 1.00 & $0.97-1.04$ \\
\hline Gender, male & $-0.11(0.24)$ & 0.90 & $0.56-1.44$ \\
\hline CIRS comorbidity score & $-0.39(0.55)$ & 0.68 & $0.23-1.99$ \\
\hline Baseline HRSD score & $-0.06(0.04)$ & 0.95 & $0.87-1.03$ \\
\hline Number of previous episodes of depression & $-0.05(0.24)$ & 0.95 & $0.60-1.51$ \\
\hline Prior treatment with antidepressants & $-0.07(0.26)$ & 0.94 & $0.56-1.56$ \\
\hline \multicolumn{4}{|l|}{ Medication } \\
\hline Dosage of sertraline & $0.52(0.39)$ & 0.59 & $0.28-1.27$ \\
\hline Non-adherence to sertraline & $-0.22(0.14)$ & 0.80 & $0.61-1.04$ \\
\hline \multicolumn{4}{|c|}{$\begin{array}{l}\text { CIRS, Cumulative IIIness Rating Scale; HR, hazard ratio; HRSD, Hamilton Rating Scale for Depression; S+NPE, sertraline plus non-progressive exercise; } S+P A E \text {, sertraline plus } \\
\text { progressive aerobic exercise. } \\
\text { a. Cox regression models: remission in the sertraline-only intervention arm is the reference category. } \\
\text { b. Unadjusted model; } \chi^{2}=8.9 \text {, d.f. }=2, P=0.01 \text {. } \\
\text { C. Adjusted model; } \chi^{2}=34.8 \text {, d.f. }=13, P=0.001 \text {. } \\
{ }^{*} P<0.05 ; * * P<0.01 \text {. }\end{array}$} \\
\hline
\end{tabular}

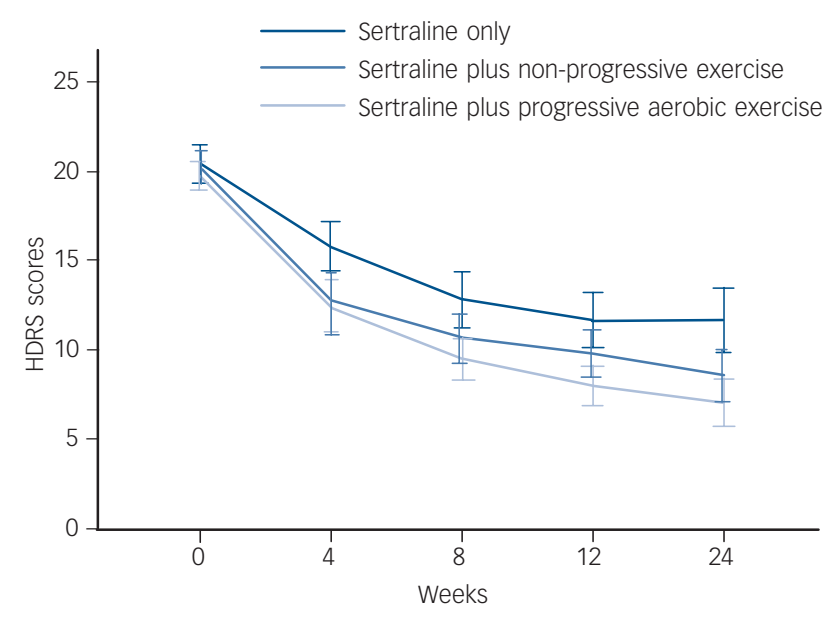

Fig. 3 Unadjusted mean scores (with 95\% confidence intervals) on the Hamilton Rating Scale for Depression (HRSD) over time.

\section{Discussion}

A significantly higher proportion of sedentary older people with major depression achieved remission with sertraline plus a 24-week exercise programme compared with those treated with sertraline alone. Both the exercise protocols were associated with earlier and higher remission rates, which were evident after only 4 weeks of treatment. The beneficial effect of physical exercise was independent of the severity and chronicity of depression, of participants' demographic characteristics and of physical comorbidities. Overall, a high degree of adherence to the interventions was observed in a frail population group that had not been sufficiently studied in this regard. ${ }^{7}$ These findings are particularly significant for clinical practice, since late-life depression is often associated with poor or slow responses to first-line pharmacotherapy, ${ }^{6,29}$ and with physical inactivity. ${ }^{30}$

\section{Other research}

To our knowledge this study is the first to examine the efficacy of structured physical exercise interventions in the antidepressant treatment of older adults with major depression. Our findings are consistent with research suggesting that exercise could exert antidepressant properties in adults with subthreshold or fully fledged clinical depression. ${ }^{8,31}$ In older patients, most commonly with non-major depression, a few studies have shown that physical exercise (group- or home-based, strength training or mixed aerobic and strength training, mostly three times per week) was effective in reducing depressive symptoms, with small to moderate effects. $^{7}$ The effect sizes of sertraline therapy alone and in combination with non-progressive exercise are larger than those recently observed. ${ }^{7}$ Several factors may account for this difference:

\begin{tabular}{|c|c|c|c|}
\hline \multirow[b]{2}{*}{ Assessment } & \multicolumn{3}{|c|}{ HRSD total score: mean (s.d.) } \\
\hline & Sertraline group $(n=42)$ & S+NPE group $(n=37)$ & S+PAE group $(n=42)$ \\
\hline Baseline & $20.4(3.4)$ & $20.1(3.2)$ & $19.8(2.6)$ \\
\hline 4 weeks & $15.8(4.4)$ & $12.7(5.1)$ & $12.4(4.8)$ \\
\hline 8 weeks & $12.8(5.0)$ & $10.7(4.1)$ & $9.6(3.9)$ \\
\hline 12 weeks & 11.7 (5.0) & $9.8(4.1)$ & $8.0(3.6)$ \\
\hline 24 weeks & 11.7 (5.9) & $8.5(4.5)$ & $7.1(4.2)$ \\
\hline
\end{tabular}


these include the use of an antidepressant drug, social support due to exercising in groups, more intense exercise protocols and a longer duration of the interventions. ${ }^{13,16,32}$ More recently another study has found that a low-intensity, low-frequency tai chi plus meditation protocol in combination with escitalopram therapy was more effective than health education meetings plus escitalopram among older volunteers with depression from the community. ${ }^{33}$ In contrast, a recent large trial failed to demonstrate significant reductions of depressive symptoms associated with physical exercise (two sessions per week) among very old people living in care homes. ${ }^{34}$ This inconsistency might be due to differences in sample characteristics (participants were more physically and cognitively impaired and only half had clinical depression in the latter study), assessment instrument (Geriatric Depression Scale), a lower frequency, intensity and adherence to the physical exercise protocol and lower antidepressant use (30\% of participants). Taken together, these findings suggest that physical exercise might need to be provided along with an antidepressant, ${ }^{33}$ or at least be scheduled with sufficient frequency and/or intensity to exert significant antidepressant effects. ${ }^{13,16}$ In our study both protocols followed the recommendations set by guidelines for physical exercise in elderly people, ${ }^{18}$ but differed in that the S+PAE intervention was characterised by increasing energy expenditure, whereas the S+NPE group exercises were aimed at improving participants' balance and strength. After adjusting for confounders, the outcomes of depression were similar in the two groups. Unlike others, ${ }^{35}$ we did not observe significant improvement in aerobic capacity in the exercise groups compared with the sertraline-only group, or between the two exercise groups. However, only a small number of participants $(n=73)$ were assessed for aerobic capacity both at baseline and after 24 weeks, so the statistical power may have not been sufficient to detect the effect of exercise on the aerobic capacity. Further analyses and larger studies should clarify whether different types of physical activity might elicit specific mechanisms of antidepressant action in elderly patients; ${ }^{13,36}$ for example, among younger adults aerobic training but not strength exercise seems associated with increases of BDNF. ${ }^{37}$

\section{Study limitations}

This study has some limitations that need to be taken into account. We did not include an active comparator, such as social support or heath education group meetings, as an augmentation strategy to sertraline. Hence, we cannot rule out that part of the additional antidepressive efficacy provided by physical exercise was related to social support rather than to exercise itself. ${ }^{32}$ However, this choice was undertaken for several reasons: first, our aim was to assess the added benefit of exercise to SSRI therapy, since SSRIs are commonly used by primary care physicians and the low efficacy of these drugs leaves many patients with continuing symptoms. ${ }^{29}$ Second, physical exercise was already shown to be more effective than comparators such as social group programmes. ${ }^{7}$ Third, patients might have been less likely to engage in non-therapeutic activities with such a high frequency. Another limitation is that participants received relatively low dosages of sertraline, despite the fact that clinicians were free to increase the dose according to the clinical indication; however, the final dosages used in our study reflect usual clinical practice. ${ }^{38}$ We were unable to control for the effect of different instructors delivering exercise interventions, as each group was trained by the same instructor in all sessions. However, we held regular meetings for the monitoring of fidelity to protocols, and we did not observe any significant between-instructor difference in participants' adherence. Despite randomisation, we observed imbalances in some baseline participant characteristics, perhaps a result of between-centre differences in patient flow. However, multivariate analyses showed that these factors did not affect the results significantly. All participants were recruited from referrals by primary care physicians. Therefore, our findings may not be generalisable to a broader population of elderly people with depression that might include individuals not seen or recognised by their physicians as depressed. Although the LOCF method is widely used, one of its limitations is the assumption that participants maintain their benefits even when they exit the study prematurely.

\section{Clinical implications}

Our findings provide the clinician with a rationale for adopting physical exercise as a potential tool in the management of late-life major depression; it is noteworthy that nearly three-quarters of the patients who received physical exercise as an add-on to sertraline therapy reached remission within the study period well beyond those who received sertraline alone. Also, remission was faster than is commonly observed for antidepressant

\begin{tabular}{|c|c|c|c|c|}
\hline Parameter & $b$ & s.e. & $95 \% \mathrm{Cl}$ & $P$ \\
\hline \multicolumn{5}{|l|}{ Fixed effects } \\
\hline Intercept & 19.87 & 0.45 & 18.99 to 20.75 & $<0.001 * *$ \\
\hline Time & -2.36 & 0.16 & -2.67 to -2.06 & $<0.001 * *$ \\
\hline Time 2 & 0.17 & 0.02 & 0.13 to 0.20 & $<0.001 * *$ \\
\hline Time 3 & -0.03 & 0.05 & -0.04 to -0.03 & $<0.001 * *$ \\
\hline $\mathrm{S}+\mathrm{NPE} \mathrm{E}^{\mathrm{a}}$ & -0.22 & 0.45 & -1.10 to 0.66 & 0.621 \\
\hline S+NPEtime & -0.46 & 0.16 & -0.77 to -0.16 & $0.003^{* *}$ \\
\hline S+NPEtime 2 & 0.05 & 0.02 & 0.01 to 0.09 & $0.007 * *$ \\
\hline S+NPEtime 3 & -0.001 & 0.0005 & -0.002 to -0.0003 & $0.010^{*}$ \\
\hline $\mathrm{S}+\mathrm{PAE} \mathrm{E}^{\mathrm{a}}$ & -0.36 & 0.43 & -1.21 to 0.49 & 0.399 \\
\hline S+PAEtime & -0.44 & 0.15 & -0.74 to -0.15 & $0.003^{* *}$ \\
\hline S+PAEtime 2 & 0.04 & 0.02 & 0.003 to 0.08 & $0.033^{*}$ \\
\hline S+PAEtime 3 & -0.01 & 0.05 & -0.002 to 0.00004 & 0.060 \\
\hline \multicolumn{5}{|l|}{ Random effects } \\
\hline Variance intercept & 8.69 & 1.57 & 6.10 to 12.37 & $<0.001 * *$ \\
\hline Variance time & 0.03 & 0.01 & 0.02 to 0.04 & $<0.001 * *$ \\
\hline ARH rho & -0.20 & 0.14 & -0.45 to 0.08 & 0.147 \\
\hline
\end{tabular}


treatment in elderly patients. ${ }^{39}$ Participants were representative of the sedentary patients with late-life major depression who are usually encountered in primary care: the vast majority had been treated with an antidepressant in the past for previous depressive episodes, but still experienced relevant - albeit mild to moderate symptoms. All these factors have been linked with worse responses to antidepressant drugs, ${ }^{40}$ and might contribute to further disability, chronicity and worse outcomes. Although in this first report we did not examine potential mechanisms and predictors of response to physical exercise, further analyses might extend knowledge on these issues and help the translation of these results to clinical practice. ${ }^{6}$ We have documented that combining physical exercise of high or low intensity with antidepressants is more effective than antidepressant drug therapy alone in older adults with major depression who are sedentary. Clinicians may consider adding exercise to the regimen of older patients with depression, people who often fail to respond to antidepressant therapy.

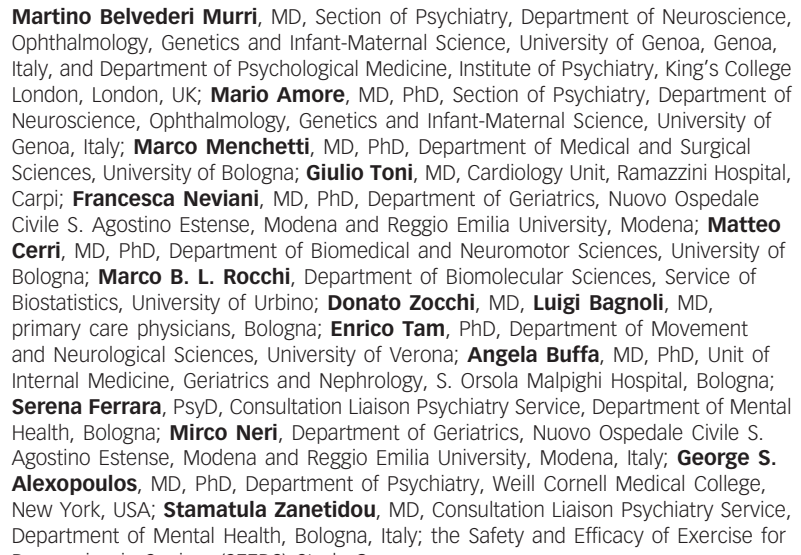

First received 28 Apr 2014, final revision 24 Oct 2014, accepted 24 Nov 2014

\section{Funding}

This research was supported by the Emilia Romagna Region University Programme (PrRU) 2010-2012 grant, Area 2 for Clinical Governance. The funders had no role in study design, data collection and analysis, decision to publish or preparation of the manuscript.

\section{Acknowledgements}

Members of the Safety and Efficacy of Exercise for Depression in Seniors (SEEDS) study group include the following. Bologna centres: Francesco Ripa di Meana, MD, Maria L. Marcaccio, MD, Paola Argnani, MD, Angelo Fioritti, MD, Mara Morini, MD, Fausto Trevisani, MD, Salvatore Squatrito, MD, Roberto Senaldi, MD, Giovanni De Girolamo, MD, PhD, Danilo MD, Salvatore Squatrito, MD, Roberto Senaldi, MD, Giovanni De Girolamo, MD, PhD, Danilo
Di Diodoro, MD, Giovanni Neri, MD, Carlo Spezia, MD, Monica Magagnoli, MD, Claudia Luciano, MD, Federica Casini, PsyD, Giuliano Ermini, MD, Piero Casarini, MD, Pier Vittorio Bardazzi, MD, Alessandro Piras MD, PhD, Giuliana Tola, Roberto Rizzo, Lorenzo De Bei and Davide Comastri. Modena-Correggio centre: Ferdinando Tripi, MD, Massimo Mannina, MD; Massimo Piepoli, MD, PhD Sandro Zoboli, Gabriele Torcianti, Niccolò Colombini, MD, PhD, Federzoni Guido, MD, Silvia Ferrari, MD, PhD, Morena Pellati, MD, Veronica Barbant Silva, MD, Agnese Cepelli, Elena Francia, PsyD, Roberto Chiesa, MD, and Anna Grazia Frignani, MD. Parma centre: Cristina Montegano, MD, PhD, Graziano Ceresini, MD, Arianna Montali, MD, Aderville Cabassi, MD, PhD, Mattia Masotti, MD, PhD, and Maria Lidia Gerra, MD.

\section{Appendix}

\section{Protocol for the non-progressive exercise intervention}

Each session of the protocol consisted of:

10 min warm-up: walking, strengthening exercises, quiet calisthenics

2 repetitions of 10 min each: mat work: stretching, calisthenics, breathing exercises
2 repetitions of 5 min each: instrumental exercises (first with a ball, then with a stick)

2 repetitions of 5 min each: balance exercises (e.g. toe walking, heel to toe, single limb stance, staggered stance)

10 min cool down: walking, quiet calisthenics

Participants were invited to rest when their heart rate exceeded the threshold of $70 \%$ of peak heart rate, or whenever they felt exhausted.

\section{Protocol for the progressive aerobic exercise intervention}

Each session of the protocol consisted of a 10 min warm-up: breathing exercises, slow cycling. This was followed by cycling at an intensity that would maintain the heart rate within the assigned target heart-rate range. Target heart rate was defined by percentage of the peak heart rate (PHR) as measured during the maximum oxygen uptake test. All exercise sessions concluded with 5-10 min of cool-down cycling.

\section{Cycling sessions}

First period (weeks 0-4): exercise bike, cycling at 60-70\% of PHR, 30-40 min Second period (weeks 5-8): treadmill exercise at 70-80\% of PHR, 40-50 min

Third period (weeks 9-12): five interval training sessions of 5 min at 85\% of PHR or 40 min of continuous treadmill at $70 \%$ of PHR

Fourth period (weeks 13-24): five interval training sessions of 6 min at $85 \%$ of PHR, or 40 min of continuous treadmill at $70 \%$ of PHR

\section{References}

1 Meijer A, Conradi HJ, Bos EH, Anselmino M, Carney RM, Denollet J, et al. Adjusted prognostic association of depression following myocardial infarction with mortality and cardiovascular events: individual patient data metaanalysis. Br J Psychiatry 2013; 203: 90-102.

2 Yohannes AM, Connolly MJ. Do antidepressants work in patients with chronic obstructive pulmonary disease with comorbid depression? Expert Rev Respir Med 2011; 5: 727-9.

3 Diniz BS, Butters MA, Albert SM, Dew MA, Reynolds CF. Late-life depression and risk of vascular dementia and Alzheimer's disease: systematic review and meta-analysis of community-based cohort studies. Br J Psychiatry 2013; 202: 329-35.

4 Alexopoulos GS. Depression in the elderly. Lancet 2005; 365: 1961-70.

5 Kennedy GJ, Marcus P. Use of antidepressants in older patients with comorbid medical conditions: guidance from studies of depression in somatic illness. Drugs Aging 2005; 22: 273-87.

6 Alexopoulos GS, Bruce ML. A model for intervention research in late-life depression. Int J Geriatr Psychiatry 2009; 24: 1325-34.

7 Bridle C, Spanjers K, Patel S, Atherton NM, Lamb SE. Effect of exercise on depression severity in older people: systematic review and meta-analysis of randomised controlled trials. Br J Psychiatry 2012; 201: 180-5.

8 Silveira H, Moraes $\mathrm{H}$, Oliveira N, Coutinho ES, Laks J, Deslandes A. Physical exercise and clinically depressed patients: a systematic review and meta-analysis. Neuropsychobiology 2013; 67: 61-8.

9 Naismith SL, Norrie LM, Mowszowski L, Hickie IB. The neurobiology of depression in later-life: clinical, neuropsychological, neuroimaging and pathophysiological features. Prog Neurobiol 2012; 98: 99-143.

10 Alexopoulos GS, Morimoto SS. The inflammation hypothesis in geriatric depression. Int J Geriatr Psychiatry 2011; 26: 1109-18.

11 Smith PJ, Blumenthal JA, Hoffman BM, Cooper H, Strauman TA, WelshBohmer $\mathrm{K}$, et al. Aerobic exercise and neurocognitive performance: a metaanalytic review of randomized controlled trials. Psychosom Med 2010; 72 : 239-52.

12 Laske C, Banschbach S, Stransky E, Bosch S, Straten G, Machann J, et al. Exercise-induced normalization of decreased BDNF serum concentration in elderly women with remitted major depression. Int I Neuropsychopharmacol 2010; 13: 595-602.

13 Brosse AL, Sheets ES, Lett HS, Blumenthal JA. Exercise and the treatment of clinical depression in adults: recent findings and future directions. Sports Med 2002; 32: 741-60 
14 Belvederi Murri M, Pariante C, Mondelli V, Masotti M, Atti AR, Mellacqua Z, et al. HPA axis and aging in depression: systematic review and metaanalysis. Psychoneuroendocrinology 2014; 41: 46-62.

15 De Vries NM, van Ravensberg CD, Hobbelen JS, Olde Rikkert MG, Staal JB, Nijhuis-van der Sanden MW. Effects of physical exercise therapy on mobility, physical functioning, physical activity and quality of life in community-dwelling older adults with impaired mobility, physical disability and/or multi-morbidity: a meta-analysis. Ageing Res Rev 2012; 11: 136-49.

16 Dunn AL, Trivedi MH, Kampert JB, Clark CG, Chambliss HO. Exercise treatment for depression: efficacy and dose response. Am J Prev Med 2005; 28: 1-8.

17 Hamilton M. Development of a rating scale for primary depressive illness. Br J Soc Clin Psychol 1967; 6: 278-96.

18 Nelson ME, Rejeski WJ, Blair SN, Duncan PW, Judge JO, King AC, et al. Physical activity and public health in older adults: recommendation from the American College of Sports Medicine and the American Heart Association. Circulation 2007: 116: 1094-105.

19 Folstein MF, Folstein SE, McHugh PR. 'Mini-mental state'. A practical method for grading the cognitive state of patients for the clinician. J Psychiatr Res 1975; 12: 189-98.

20 Agostoni P. Cardiopulmonary exercise testing for heart failure patients: a hodgepodge of techniques, parameters and interpretations. In other words, the need for a time-break. Eur Heart J 2006; 27: 633-4.

21 Guy W. ECDEU Assessment Manual for Psychopharmacology. U.S. Dept. of Health, Education, and Welfare, Public Health Service, Alcohol, Drug Abuse and Mental Health Administration, NIMH Psychopharmacology Research Branch, Division of Extramural Research Programs, 1976.

22 Muijsers RB, Plosker GL, Noble S. Spotlight on sertraline in the management of major depressive disorder in elderly patients. CNS Drugs 2002; 16: 789-94.

23 Vigorito C, Giallauria F. Effects of exercise on cardiovascular performance in the elderly. Front Physiol 2014; 5: 51.

24 Muscari A, Giannoni C, Pierpaoli L, Berzigotti A, Maietta P, Foschi E, et al. Chronic endurance exercise training prevents aging-related cognitive decline in healthy older adults: a randomized controlled trial. Int J Geriatr Psychiatry 2010; 25: 1055-64.

25 Sheehan DV, Lecrubier $\mathrm{Y}$, Sheehan $\mathrm{KH}$, Amorim P, Janavs J, Weiller $\mathrm{E}$, et al. The Mini-International Neuropsychiatric Interview (M.I.N.I.): the development and validation of a structured diagnostic psychiatric interview for DSM-IV and ICD-10. J Clin Psychiatry 1998; 59 (suppl 20): 22-33.

26 Miller MD, Paradis CF, Houck PR, Mazumdar S, Stack JA, Rifai AH, et al. Rating chronic medical illness burden in geropsychiatric practice and research: application of the Cumulative IIIness Rating Scale. Psychiatry Res 1992; 41: 237-48.
27 Craig $C L$, Marshall $A L$, Sjostrom $M$, Bauman $A E$, Booth $M L$, Ainsworth $B E$, et al. International physical activity questionnaire: 12-country reliability and validity. Med Sci Sports Exerc 2003; 35: 1381-95.

28 Shek DT, Ma CM. Longitudinal data analyses using linear mixed models in SPSS: concepts, procedures and illustrations. ScientificWorldJournal 2011; 11: $42-76$.

29 Alexopoulos GS, Katz IR, Reynolds CF, Carpenter D, Docherty JP, Ross RW. Pharmacotherapy of depression in older patients: a summary of the expert consensus guidelines. J Psychiatr Pract 2001; 7: 361-76.

30 Dillon C, Machnicki G, Serrano CM, Rojas G, Vazquez G, Allegri RF. Clinical manifestations of geriatric depression in a memory clinic: toward a proposed subtyping of geriatric depression. J Affect Disord 2011; 134: 177-87.

31 Conn VS. Depressive symptom outcomes of physical activity interventions: meta-analysis findings. Ann Behav Med 2010; 39: 128-38.

32 Cho KL. Effect of Tai Chi on depressive symptoms amongst Chinese older patients with major depression: the role of social support. Med Sport Sci 2008; 52: 146-54.

33 Lavretsky H, Alstein LL, Olmstead RE, Ercoli LM, Riparetti-Brown M, Cyr NS et al. Complementary use of tai chi augments escitalopram treatment of geriatric depression: a randomized controlled trial. Am J Geriatr Psychiatry 2011; 19: 839-50

34 Underwood M, Lamb SE, Eldridge S, Sheehan B, Slowther AM, Spencer A, et al. Exercise for depression in elderly residents of care homes: a cluster-randomised controlled trial. Lancet 2013; 382: 41-9.

35 Blumenthal JA, Babyak MA, Moore KA, Craighead WE, Herman S, Khatri $P$, et al. Effects of exercise training on older patients with major depression. Arch Intern Med 1999; 159: 2349-56.

36 Voss MW, Heo S, Prakash RS, Erickson KI, Alves H, Chaddock L, et al. The influence of aerobic fitness on cerebral white matter integrity and cognitive function in older adults: results of a one-year exercise intervention. Hum Brain Mapp 2013; 34: 2972-85.

37 Huang T, Larsen KT, Ried-Larsen M, Moller NC, Andersen LB. The effects of physical activity and exercise on brain-derived neurotrophic factor in healthy humans: a review. Scand J Med Sci Sports 2014; 24: 1-10.

38 Sheikh Jl, Cassidy EL, Doraiswamy PM, Salomon RM, Hornig M, Holland PJ, et al. Efficacy, safety, and tolerability of sertraline in patients with late-life depression and comorbid medical illness. J Am Geriatr Soc 2004; 52: 86-92.

39 Zivin K, Kales HC. Adherence to depression treatment in older adults: a narrative review. Drugs Aging 2008; 25: 559-71.

40 Calati R, Salvina SM, Balestri M, Marsano A, De Ronchi D, Aguglia E, et al. Antidepressants in elderly: metaregression of double-blind, randomized clinical trials. J Affect Disord 2013; 147: 1-8.

\section{0 words}

\section{Authenticity}

\section{David Brunskill}

Four components determine the degree of authenticity: awareness that we all have good and bad aspects; unbiased processing of both these aspects that should be accepted; behaviour congruent with the true self; and relational orientation in which there is honesty/self-disclosure that allows others to see us as we really are. Striving for a high degree of authenticity can seem a lofty aim (think Maslow's pyramid) because to achieve it, one's core self must first be identified, then accepted and finally, allowed to live and be. Perhaps the psychological prize of healthy functioning and life satisfaction is worth an inevitable struggle? 